\title{
A Research of Dynamic RCS for Aircrafts under Complex Maneuvering
}

\author{
Ya-Lin Huang ${ }^{+}$, Chen-Xin Zhang, Gao-Xiang Zou, Ming-Cai Sun \\ Air and Missile Defense College, Air Force Engineering University, Xi' an 710051, China
}

\begin{abstract}
The flight attitude change is a vital factor for the RCS (Radar Cross Section) fluctuation of moving targets. A method of dynamic RCS calculation for aircrafts under typical maneuvering is proposed in this paper. Firstly, the all-space static RCS database is built according to the CAD model of aircrafts. Secondly, three typical maneuverings are established according to the theory of aerodynamics. Then the attitude angles the track established are calculated including azimuth and elevation angles. Finally, the dynamic RCS data are obtained by the calculated attitude angles of the track corresponding to that of allspace static RCS database. Because the static RCS database is under repeated check and amendment with tested data, the dynamic RCS data simulated are precise enough for various research based on dynamic RCS.
\end{abstract}

Keywords: typical maneuvering; dynamic RCS; attitude angles.

\section{Introduction}

In order to improve the capability for radar to confront with advanced stealth fighter, the anti-stealth comes into being along with the development of stealth technology [1]. One of the significant intersections between them lies in the control for the RCS. It is not easy to get the RCS data for moving targets due to variety of reasons such as high cost and technology limit [2]. For all this, there are still two ways to arrive. Usually we can gain dynamic RCS from the RCS test field which can provide us precise data for research [3]. However, it is useless when meeting with non-cooperative targets. Besides, we can also get it by electromagnetic simulation which is more convenient and feasible under certain precision [4].

There have been lots of achievements about dynamic RCS prediction over the years. But for the RCS of aircraft under complex maneuvering approaching practical flight, there are a lot left to do. The research on dynamic RCS is a combination of electromagnetic calculation and track planning [5]. There are some explorations about this. Dai et al proposed a simulation method of dynamic RCS for typical maneuvering targets [6]. Zhuang et al proposed a novel dynamic RCS simulation approach for level flight aircraft considering the vibration in the track [7]. Besides, Liu combines the Monte Carlo simulation with graphical electromagnetics computing (GRECO) to analyze the influence of vibration on dynamic RCS [8]. In order to get the RCS for aircrafts approaching to practical flight effect, the precise flight track must be established. According to the theory of aerodynamics, the complex maneuvering track on some given suppose is set up. At the same time, a static RCS database is established where each RCS value corresponds to a set of attitude angles. Finally, the dynamic RCS data can be gotten by the corresponding attitude angles between calculated attitude angles and those of static database. Because the static RCS data cannot embody all the calculated attitude angles, the double bilinear interpolation method is adopted to select reasonable attitude angles to confirm the dynamic RCS data eventually.

\section{Static RCS Database Establishment}

\footnotetext{
${ }^{+}$Corresponding author. Tel.: Ya-lin Huang, 18629584315.

E-mail address: hyl_940102@163.com.
} 


\subsection{Definition on Target Coordinate}

As shown in picture 1, the origin of target coordinate lies in centroid of aircrafts. The axis OX points to the nose along the fuselage. The axis OZ lies in the symmetrical plane of fuselage. The axis OY is perpendicular to the symmetrical plane of fuselage and its direction is finally confirmed by the right-hand crew rule. After stipulating the coordinate axis, the attitude angle can be confirmed then. The azimuth angle is the included angle between axis $\mathrm{OX}$ and plane $\mathrm{OXY}$ in the sight of radar which varies from $0^{\circ}$ to $360^{\circ}$. $0^{\circ}$ is along the nose of aircraft. $180^{\circ}$ is along the tail. The elevation angle is the included angle between sight of radar and plane XOY which varies from $0^{\circ}$ to $180^{\circ}$. $0^{\circ}$ is right above the back of aircrafts. $180^{\circ}$ is right below the belly of aircrafts.

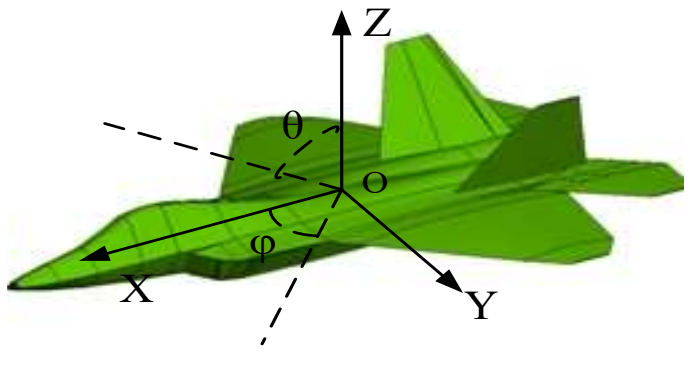

Fig. 1: Coordinate Definition

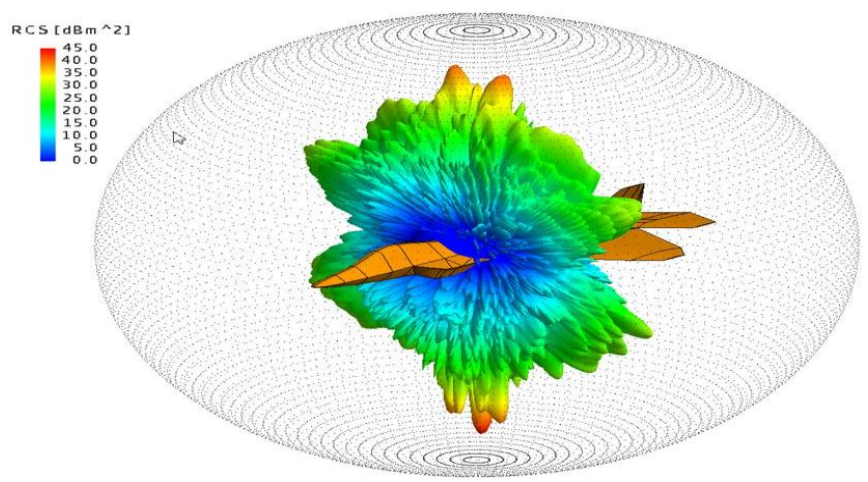

Fig. 2: All-Space Static RCS Database

\subsection{Static RCS Database}

After establishing the CAD model for an aircraft, it is imported into electromagnetic software. Then the model is under subdivision and solution settings. There are many solutions to choose such as MoM (Method of Moment), PO (Physical Optics), FDTD (Finite Different Time Domain) and so on. Because the database is of large amount of calculations, the PO method is chosen. PO is a high frequency approximation method which is efficient especially for electrically large size targets. The polarization is another factor that has a significant effect on the fluctuation of RCS. In this paper, the HH polarization is selected and the frequency is set at $1 \mathrm{GHz}$. After all the calculation settings finished, the all-space $\left(0^{\circ}<\theta<180^{\circ}, 0^{\circ}<\varphi<360^{\circ}\right)$ database is calculated at the step of $1^{\circ}$ and all $181 \times 361$ groups of data are under calculation. In order to ensure the precision of the database, all the RCS data are repeatedly checked with tested data. The result is shown in picture 2 .

\section{Complex Maneuvering Track Establishment}

\subsection{Aerodynamics Theory}

Before setting up the track, the attribute of aircraft must be confirmed [9]. Aircrafts that applied into track planning can be divided into two types: the 3 Degree-OF-Freedom (DOF) and 6 DOF model. The 3 DOF model considers aircrafts as particle. It is a simplified model that only consists of formula of force and centroid. The 6 DOF model regards aircrafts as rigid body. It comprehensively describes most characteristics including the formula of force, centroid, moment and attitude angles. In this paper, we take the 6 DOF model to establish the maneuvering track for a more accurate flight effect. The dynamical equation of 6 DOF model is shown as Eq. (1):

$$
m \bullet\left(\left[\begin{array}{c}
\dot{V}_{x} \\
\dot{V}_{y} \\
\dot{V_{z}}
\end{array}\right]+\left[\begin{array}{ccc}
0 & -w_{z} & w_{y} \\
w_{z} & 0 & -w_{x} \\
-w_{y} & w_{x} & 0
\end{array}\right] \bullet\left[\begin{array}{c}
V_{x} \\
V_{y} \\
V_{z}
\end{array}\right]\right)=\left[\begin{array}{c}
F_{x} \\
F_{y} \\
F_{z}
\end{array}\right]-\left[\begin{array}{c}
\sin \vartheta \\
\cos \vartheta \cos \gamma \\
-\cos \vartheta \sin \gamma
\end{array}\right] \bullet G
$$

In Eq. (1), $\mathrm{m}$ is the mass of the aircraft. $\mathrm{G}$ is the gravity. $V_{x}, V_{y}, V_{z}$ are respectively the projection of velocity vector on axis $\mathrm{X}, \mathrm{Y}$ and Z. $F_{x}, F_{y}, F_{z}$ are respectively the projection of the resultant force except $\mathrm{G}$ on axis $\mathrm{X}, \mathrm{Y}$ and $\mathrm{Z} . w_{x}, w_{y}, w_{z}$ are respectively the projection of angular velocity on axis $\mathrm{X}, \mathrm{Y}$ and $\mathrm{Z}$. $\vartheta$ is the pitching angle. $\gamma$ is the roll angle.

The mass center motion equation is shown as Eq. (2): 


$$
\left[\begin{array}{c}
\dot{x} \\
\dot{y} \\
\dot{z}
\end{array}\right]=L_{d t} \bullet\left[\begin{array}{c}
V_{x} \\
V_{y} \\
V_{z}
\end{array}\right]
$$

The $L_{d t}$ can be described as Eq. (2):

$$
L_{d t}=\left[\begin{array}{ccc}
\cos \psi \cos \vartheta & \sin \psi \sin \gamma-\cos \psi \cos \gamma & \sin \psi \cos \gamma+\cos \psi \sin \vartheta \sin \gamma \\
\sin \vartheta & \cos \vartheta \cos \gamma & -\cos \vartheta \sin \gamma \\
-\sin \psi \cos \vartheta & \cos \psi \sin \gamma+\sin \psi \sin \vartheta \cos \gamma & \cos \psi \cos \gamma-\sin \psi \sin \vartheta \sin \gamma
\end{array}\right]
$$

In Eq. (2) and (3), (x, y, z) are the orthogonal coordinate in the radar coordinate. $\psi$ is the yaw angle. $\vartheta$, "It and $\psi$ are collectively known as Euler angles [10].

\subsection{Complex Track Establishment}

The complex maneuvering for aircrafts is usually a combination of some basic motions. At the same time, the track planning for aircrafts is always on some specific purpose especially for military aircrafts. In this section, two practical tracks are established including track I and track II. They are shown in picture 3 and 4.

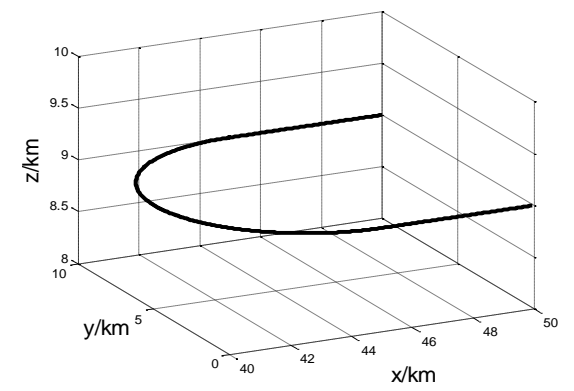

Fig. 3: Track I

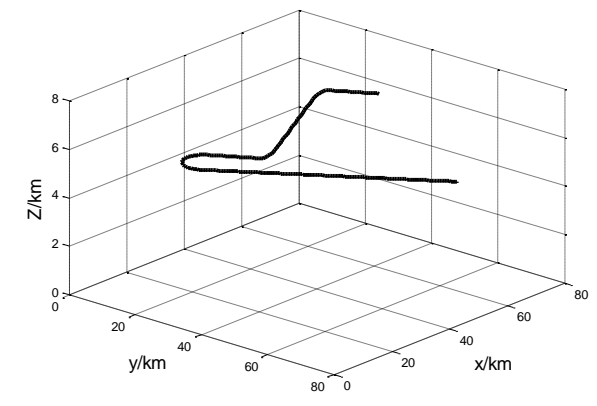

Fig. 4: Track II

The track I is set for aircrafts encountering with danger when performing tasks asking for a way to get rid of threat. The track II is set for aircrafts that has finished tasks seeking for swift escaping.

\section{Dynamic RCS Calculation}

\subsection{Coordinate Transformation}

After establishing the complex track, the orthogonal coordinate in the track can be obtained. Because the static RCS database corresponds to the attitude angles in radar coordinate, coordinate transformation must be carried out. The transformation equation between orthogonal coordinate and polar coordinate (attitude angles) can be shown in Eq. 4.

$$
\left\{\begin{array}{l}
\varphi(t)=\arctan \frac{y_{T}(t)}{x_{T}(t)} \\
\theta(t)=\arctan \frac{z_{T}(t)}{r(t)}
\end{array}\right.
$$

In Eq. $5,\left(x_{T}(t), y_{T}(t), z_{T}(t)\right)$ is the aircrafts' track point in target coordinate. $\varphi(t)$ is the azimuth angle. $\theta(t)$ is the elevation angle. $r(t)$ is the radius in the polar coordinate which can be shown in Eq. 5 .

$$
r(t)=\sqrt{\left(x_{T}(t)\right)^{2}+\left(y_{T}(t)\right)^{2}+\left(z_{T}(t)\right)^{2}}
$$

The attitude angles change of track I and Track II can be shown in picture 5 and 6 . 


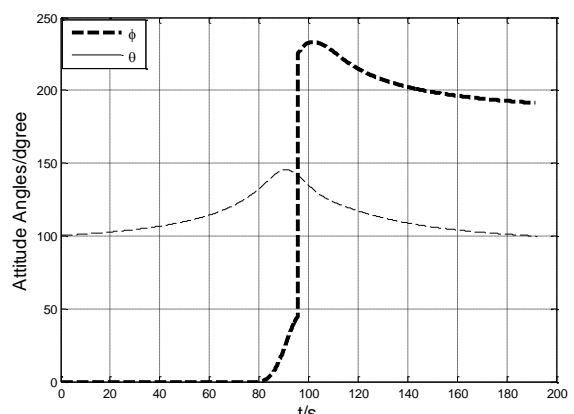

Fig. 5: Attitude Angles Variation of Track I

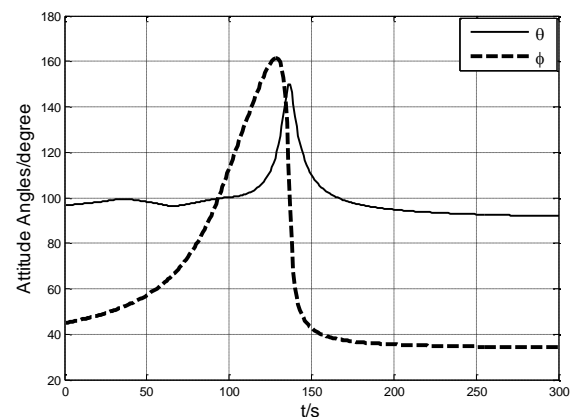

Fig. 6: Attitude Angles Variation of Track II

\subsection{Double Bilinear Interpolation Method}

Because the capacity limit of static database, it cannot cover with all the attitude angles calculated. So the double bilinear interpolation method is applied to solve the problem. The key part of the double bilinear interpolation method is to seek for reasonable attitude angles value around the calculated attitude angle value in the static database. The details are shown in picture 7.

$$
\begin{gathered}
\sigma\left(\varphi_{L}, \theta_{G}\right) \cdots-\sigma\left(\varphi, \theta_{L}\right) \cdots\left(\varphi_{G}, \theta_{G}\right) \\
\sigma(\varphi, \theta) \\
\sigma\left(\varphi_{L}, \theta_{L}\right) \ldots\left(\varphi, \theta_{G}\right) \cdots\left(\varphi_{G}, \theta_{L}\right)
\end{gathered}
$$

Fig 7 Operation of Double Bilinear Interpolation Method

In figure 7, $\varphi$ and $\theta$ are respectively the calculated azimuth angle and elevation angle from established track. $\varphi_{G}, \theta_{G}$ are respectively greater than $\varphi$ and $\theta$ in static database. At the same time, they are also the nearest one. In a similar way, $\varphi_{L}$ and $\theta_{L}$ are respectively less than that in static database which are also the nearest one. Firstly, the interpolation is applied in the azimuth dimension. The details can be shown in Eq. 6 and Eq. 7.

$$
\begin{aligned}
& \sigma\left(\varphi, \theta_{L}\right) \approx \frac{\varphi_{G}-\varphi}{\varphi_{G}-\varphi_{L}} \bullet \sigma\left(\varphi_{L}, \theta_{L}\right)+\frac{\varphi-\varphi_{L}}{\varphi_{G}-\varphi_{L}} \bullet \sigma\left(\sigma_{G}, \varphi_{L}\right) \\
& \sigma\left(\varphi, \theta_{G}\right) \approx \frac{\varphi_{G}-\varphi}{\varphi_{G}-\varphi_{L}} \bullet \sigma\left(\varphi_{L}, \theta_{G}\right)+\frac{\varphi-\varphi_{L}}{\varphi_{G}-\varphi_{L}} \bullet \sigma\left(\sigma_{G}, \varphi_{G}\right)
\end{aligned}
$$

Then the interpolation is applied in elevation dimension. The details can be shown in Eq 8 .

$$
\sigma(\varphi, \theta) \approx \frac{\theta_{G}-\theta}{\theta_{G}-\theta_{L}} \bullet \sigma\left(\varphi, \theta_{L}\right)+\frac{\theta-\theta_{L}}{\theta_{G}-\theta_{L}} \bullet \sigma\left(\varphi, \theta_{G}\right)
$$

By means of double bilinear interpolation, we can finally obtain the reasonable attitude angles corresponding to the static database.

\subsection{Dynamic RCS Extraction}

Based on the corresponding attitude angles between calculated attitude angles and that of the static database, we can extract dynamic RCS for the established track. The dynamic RCS for track I and track II can be shown in picture 9 .

In picture 8 and 9, the RCS fluctuates with time in track I and II are given. In track I, the RCS fluctuates dramatically when aircrafts turn around. It is similar when diving motion appears. That's because the unconventional motions often expose the un-stealth parts around aircrafts. The dynamic RCS data simulated are precise enough for various research based on dynamic RCS because the method of establishing static RCS database is under repeatedly check with tested data. 


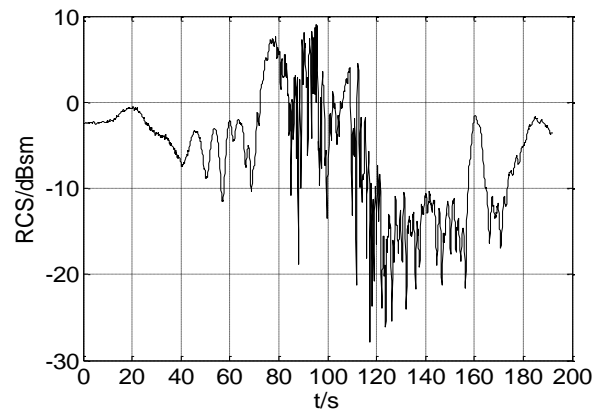

Fig 8: Dynamic RCS for Track I

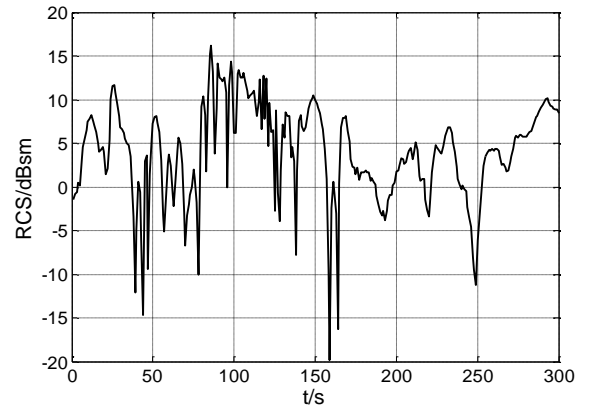

Fig 9: I Dynamic RCS Track II

\section{Conclusion}

In order to research the dynamic RCS for aircrafts under complex maneuvering, a novel approach is proposed in this paper. According to the aerodynamics, two complex maneuvering tracks on some given purpose are set up. At the same time, an all-space static RCS database is established by the electromagnetic software. Finally, the dynamic RCS data are eventually obtained by the bridge of attitude angles. After calculating the attitude angles from the established complex track, the dynamic RCS can be eventually extracted from the static RCS database. This work is useful for both survivability of aircrafts and detection of radar. Besides, the work can be applied to the track planning when material parameters are provided. In a word, the prediction of moving targets' RCS is useful in modern military application. Also, the establishment of tracks should take the performances of aircrafts and the aeronautics theory in moving process into consideration. So the future work should combine more details in moving process in order to get more precise results.

\section{Reference}

[1] H. Mehri, A. Abdolali, M. Fallah et al. "A new method for optimizing RCS of the nose of flying objects," Microwave and Optical Technology Letters, 2015,57(6): 1361-1365.

[2] C. Dai, Z. H. Xu, and S. P. Xiao, "Analysis for difference between dynamic RCS and Static RCS characteristics of radar target," Journal of Signal Processing, 2013,29(9): 1256-1263.

[3] C. F. Hu, N. J. Li, W. J. Chen, et al. "High-precision RCS measurement of aircraft's weak scattering source," Chinese Journal of Aeronautics, 2016, 29(3): 772-778.

[4] B. Persson and M. Norsell. "On modeling RCS of aircraft for flight simulation," IEEE Antennas and Progation Magzine, 2014, 56(4):34-43.

[5] Q. Yan, J. J. Jiang, S. M. You. "Real-time programming method for flight path of unmanned vehicle based on dynamic RCS," Journal of Beijing University of Aeronautics and Astronautics, 2011, 37(9): 1115-1121.

[6] C. Dai, Z. H. Xu, S. P. Xiao. "Simulation method of dynamic RCS for non-cooperative targets," Acta Aeronautics et Astronautics Sinica, 2014, 35(5):1374-1384.

[7] Y. Q. Zhuang, C. X. Zhang, X. K. Zhang. "A novel simulation approach of aircraft dynamic RCS," Progress In Electromagnetics Research M, 2014, 36: 85-91.

[8] J. Liu, N. Fang, B. F. Wang, et al. "A novel dynamic RCS simulation and analysis method considering attitude perturbation," Journal of Electromagnetic Waves and Application, 2015, 29(14): 1841-1858.

[9] J. Z. Ji, C. Y. Shu and P. L. Huang. "Application of Euler angles in flight path simulation," Journal of Beijing University of Aeronautics and Astronautics. 2014, 46(2): 218-224.

[10] Q. Yan, J. J. Xiong, S. M. You. "Real-time programming method for flight path of unmanned vehicle based on dynamic RCS," Journal of Beijing University of Aeronautics and Astronautics. 2011, 37(9): 1115-1121 